\title{
Delay and Power Expressions for a CMOS Inverter Driving a Resistive-Capacitive Load
}

\author{
Victor Adler and Eby G. Friedman \\ Department of Electrical Engineering \\ University of Rochester \\ Rochester, NY 14627 \\ adler@ee.rochester.edu,friedman@ee.rochester.edu
}

\begin{abstract}
A delay and power model of a CMOS inverter driving a resistive-capacitive load is presented. The model is derived from Sakurai's alpha power law and exhibits good accuracy. The model can be used to design and analyze those inverters that drive a large $R C$ load when considering both speed and power. Expressions are provided for estimating the propagation delay, transition time, and short circuit power dissipation for a CMOS inverter driving resistive-capacitive interconnect lines.
\end{abstract}

\section{Introduction}

As the size of CMOS integrated circuits continues to increase, interconnections have become increasingly significant. With a linear increase in length, interconnect delay increases quadratically due to a linear increase in both interconnect resistance and capacitance [1]. Also, large interconnect loads not only affect performance but cause excess power to be dissipated. A large $R C$ load degrades the waveform shape, dissipating excessive short circuit power in the following stages loading a CMOS logic gate.

Several methods have been introduced to reduce interconnect delay so that these impedances do not dominate the delay of a critical path [1-4]. Furthermore, with the introduction of portable computers, power has become an increasingly important factor in the circuit design process. Thus, power consumption must be accurately estimated when considering techniques for improving the speed of long interconnections.

This research was supported in part by the National Science Foundation under Grant No. MIP-9208165 and Grant. No. MIP-9423886, the Army Research Office under Grant No. DAAH04-93-G0323, and by a grant from the Xerox Corporation.
In this paper, an analytical expression for the transient response of a CMOS inverter driving a lumped $R C$ load is presented. The approach is different from Kayssi et al. [5] in that a lumped $R C$ load is considered rather than a lossless capacitive load. Furthermore, Sakurai's alpha power law [6] is used to describe the circuit operation of the CMOS transistors rather than the classical Shichman-Hodges model [7]. The alpha power law model considers short channel behavior, permitting increased accuracy and generality in the delay and power expressions. These expressions are used to estimate the propagation delay and the rise and fall times (or transition times) of a CMOS inverter. Since the output waveform is accurately calculated, the short circuit power dissipated by the following stage can also be estimated. Furthermore, due to its relative simplicity, this expression permits linear programming techniques to be used when optimizing the placement of buffers for both speed and power.

The paper is organized as follows: expressions for an inverter driving a lumped $R C$ load are derived, and characteristic delay equations are presented and compared with SPICE in Section II. Error bounds are discussed in Section III. In Section IV, the short circuit power dissipation of a CMOS inverter following a lumped $R C$ load is introduced and compared with SPICE. Finally, some concluding remarks are offered in Section V.

\section{General Solution}

An analytical expression describing the behavior of an inverter driving a lumped $R C$ load (shown in Figure 1) based on Sakurai's alpha power law model [6] is presented. The alpha power law model more accurately describes short-channel behavior, such as velocity saturation, while providing a tractable equa- 


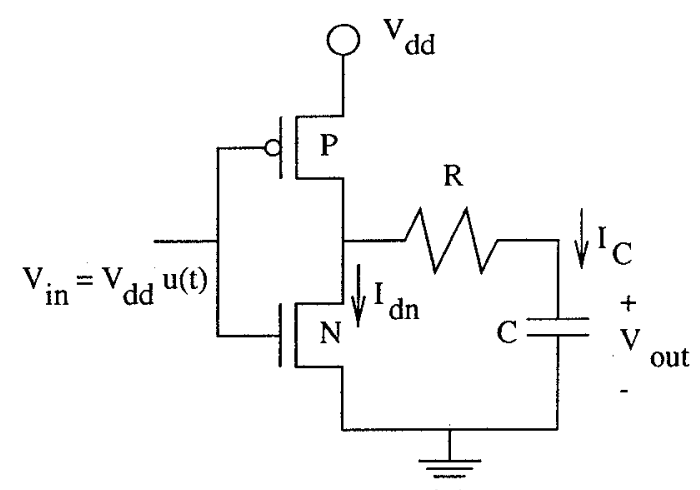

Figure 1. CMOS inverter driving an $R C$ load

tion. Specifically, the linear region of the ON transistor is used, since a large portion of the circuit operation occurs within this region under the assumption of a step input signal. When the input to the inverter is a unit step or fast ramp, $V_{\text {out }}$ is initially larger than $V_{G S}-V_{T}$ for a shorter period of time than if the input to the inverter is a slow ramp. Therefore, the circuit operates in the linear region for a greater portion of the total transition time for a large $R C$ load. If the input waveform increases more slowly or the load impedance is small, the inverter operates in the saturation region for a longer time before switching into the linear region.

Only the falling output (rising input) waveform is considered. The following analysis, however, is equally applicable to a rising output (falling input) waveform. The lumped load is modeled as a resistor in series with a capacitor. The current through the output load capacitance is the same magnitude and opposite sign as the $\mathrm{N}$-channel drain current. The capacitive current is

$$
i_{C}=C \frac{d V_{o u t}}{d t}=-i{ }_{d}
$$

where $C$ is the output capacitance, $V_{\text {out }}$ is the voltage across that capacitance, $i_{C}$ is the current through the capacitor, and $i_{d}$ is the drain current through the $\mathrm{N}$ channel device.

The N-channel linear drain current is given by

$$
\begin{gathered}
-C \frac{d V_{\text {out }}}{d t}=i_{d}=\frac{I_{d o}}{V_{d o}}\left(\frac{V_{g s}-V_{T}}{V_{D D}-V_{T}}\right)^{\alpha} V_{d s}, \\
\text { for } V_{g s} \geq V_{T}, V_{g s}-V_{T} \geq V_{d s} .
\end{gathered}
$$

In the alpha power law model, $I_{d o}$ represents the drive current of the MOS device and is proportional to W/L,
$V_{d o}$ represents the drain-to-source voltage at which velocity saturation occurs with $V_{G S}=V_{D D}$ and is a process dependent constant, and $\alpha$ models the process dependent degree to which the velocity saturation affects the drain-to-source current. $\alpha$ is within the range $1 \leq \alpha \leq 2$, where $\alpha=1$ corresponds to a device operating strongly under velocity saturation, while $\alpha=2$ represents a device where there is negligible velocity saturation. $V_{D D}$ is the supply voltage, and $V_{T}$ is the MOS threshold voltage (where $\mathrm{V}_{\mathrm{TN}}\left(\mathrm{V}_{\mathrm{TP}}\right)$ is the $\mathrm{N}$-channel (P-channel) threshold voltage).

Assuming a unit step input is applied to the circuit shown in Figure 1, $V_{\text {out }}$ can be derived from (2). The linear equation, rewritten in Laplace form, is

$$
\begin{aligned}
S C V_{\text {out }}+S \mho_{d o} R C V_{o u t}+\mho_{d o} V_{\text {out }} & = \\
C V_{o}(0)+\mho_{d o} R C V_{\text {out }}(0) &
\end{aligned}
$$

where $\mho_{d o}=\frac{I_{d o}}{V}$ is the saturation conductance.

Equation (3) yields

$$
V_{\text {out }}(t)=V_{\text {out }}(0) e^{\frac{-U_{d o}}{\mho_{d o} R C+\frac{C}{K}} t} .
$$

When $K=2$, (4) closely approximates SPICE. This empirical value for $K$ is stable for a wide range of $R C$ loads and across several short-channel technologies. Graphs of $V_{\text {out }}$ versus time for several values of resistance and capacitance are shown in Figure 2. The analytical expression shown in (4) closely approximates SPICE for most of the region of operation for a range of load impedances from $10 \Omega$ to $1000 \Omega$ and from $10 \mathrm{fF}$ to $1 \mathrm{pF}$. The maximum error over this range, shown in Table $\mathrm{I}$, is $27 \%$ for a $.8 \mu \mathrm{m}$ CMOS technology.

From (4), the propagation delay of a CMOS inverter calculated at the $50 \%$ point $t_{P D}$ is

$$
t_{P D}=.693 \frac{\frac{C}{K}+\mho_{d o} R C}{\mho_{d o}} .
$$

The transition time of a CMOS inverter driving a lumped $R C$ load calculated at the $90 \%$ point $t_{t}$ is

$$
t_{t}=2.3 \frac{\frac{C}{K}+\mho_{d o} R C}{\mho_{d o}}
$$



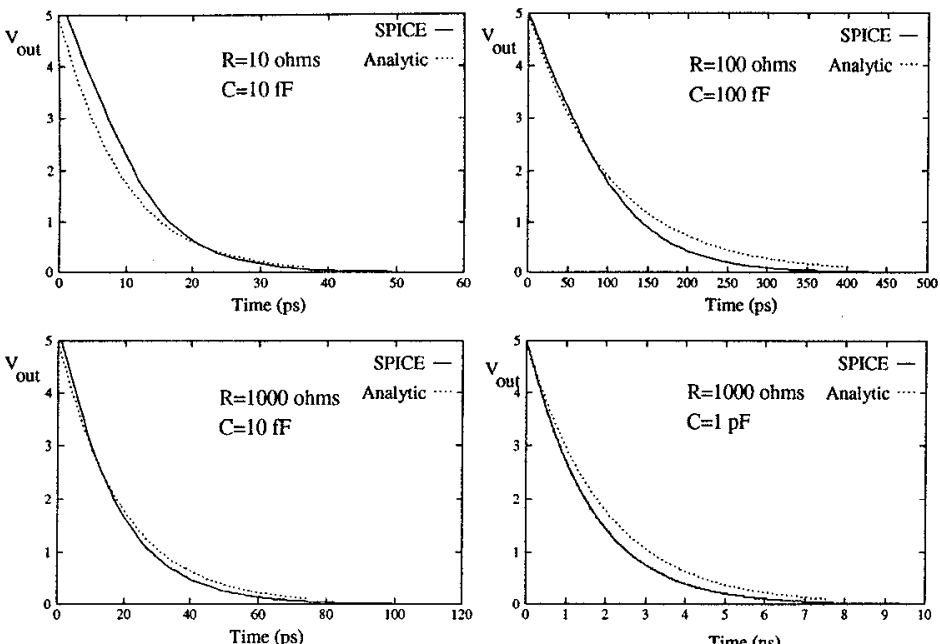

Figure 2. Output response of a

CMOS inverter driving an $R C$ load

The accuracy of the analytic model versus SPICE is tabulated in Table I for a wide variety of output load resistances and capacitances. Note that both the $50 \%$ propagation delay and the transition time are shown. The maximum error of the transition time $t_{t}$ as compared with SPICE is $27 \%$, and the maximum error of the propagation delay $t_{P D}$ as compared with SPICE is $25 \%$ for a $.8 \mu \mathrm{m}$ CMOS technology.

Equations (5) and (6) can be used to estimate the propagation delay and transition time of a CMOS inverter driving a long resistive interconnect line. Since the shape of the output waveform is known, these expressions can also be used to estimate the short circuit power dissipation of the CMOS gate loading the high impedance interconnect line, as described in Section IV.

Table I. Propagation delay and rise time of an inverter driving an $R C$ load (.8 $\mu \mathrm{m}$ CMOS technology).

\begin{tabular}{|c|c|c|c|c|c|c|c|}
\hline $\begin{array}{c}\text { Load } \\
\text { Resis- } \\
\text { tance }\end{array}$ & $\begin{array}{c}\text { Load } \\
\text { Capaci- } \\
\text { tance }\end{array}$ & Analytic & SPICE & Analytic & SPICE & $t_{t}$ & $t_{P D}$ \\
\cline { 3 - 8 } & & & & & \multicolumn{2}{|c|}{$t_{t}$} & \\
\hline $10 \Omega$ & $.01 \mathrm{pF}$ & $21 \mathrm{ps}$ & $22 \mathrm{ps}$ & $6.5 \mathrm{ps}$ & $8.7 \mathrm{ps}$ & $4 \%$ & $25 \%$ \\
\hline $10 \Omega$ & $.1 \mathrm{pF}$ & $215 \mathrm{ps}$ & $176 \mathrm{ps}$ & $65 \mathrm{ps}$ & $70 \mathrm{ps}$ & $22 \%$ & $7 \%$ \\
\hline $10 \Omega$ & $1 \mathrm{pF}$ & $2.2 \mathrm{~ns}$ & $1.7 \mathrm{~ns}$ & $649 \mathrm{ps}$ & $680 \mathrm{ps}$ & $27 \%$ & $4 \%$ \\
\hline $100 \Omega$ & $.01 \mathrm{pF}$ & $24 \mathrm{ps}$ & $22 \mathrm{ps}$ & $7.2 \mathrm{ps}$ & $8.8 \mathrm{ps}$ & $6 \%$ & $19 \%$ \\
\hline $100 \Omega$ & $.1 \mathrm{pF}$ & $235 \mathrm{ps}$ & $187 \mathrm{ps}$ & $71 \mathrm{ps}$ & $73 \mathrm{ps}$ & $25 \%$ & $2 \%$ \\
\hline $100 \Omega$ & $1 \mathrm{pF}$ & $2.4 \mathrm{~ns}$ & $1.9 \mathrm{~ns}$ & $712 \mathrm{ps}$ & $711 \mathrm{ps}$ & $25 \%$ & $0 \%$ \\
\hline $1000 \Omega$ & $.01 \mathrm{pF}$ & $44 \mathrm{ps}$ & $39 \mathrm{ps}$ & $13 \mathrm{ps}$ & $13 \mathrm{ps}$ & $13 \%$ & $0 \%$ \\
\hline $1000 \Omega$ & $.1 \mathrm{pF}$ & $444 \mathrm{ps}$ & $365 \mathrm{ps}$ & $133 \mathrm{ps}$ & $115 \mathrm{ps}$ & $15 \%$ & $16 \%$ \\
\hline $1000 \Omega$ & $1 \mathrm{pF}$ & $4.4 \mathrm{~ns}$ & $3.6 \mathrm{~ns}$ & $1.3 \mathrm{~ns}$ & $1.1 \mathrm{~ns}$ & $22 \%$ & $18 \%$ \\
\hline
\end{tabular}

\section{Error Bounds}

The maximum error for the transition time for $R C$ loads ranging from $10 \Omega$ to $1000 \Omega$ and 10 $\mathrm{fF}$ to $1 \mathrm{pF}$ and for three different technologies (.8 $\mu \mathrm{m}, 1.2 \mu \mathrm{m}$, and $1.6 \mu \mathrm{m} \mathrm{CMOS})$ is $57 \%$. The maximum error for the propagation delay is $43 \%$ over the same ranges and technologies. As the capacitance increases to $1 \mathrm{pF}$, the error of the propagation delay generally decreases to less than $20 \%$. A similar decrease occurs for the transition time. Furthermore, both errors generally decrease with increasing load resistance.

The improved accuracy with increasing load resistance and capacitance is due to the effect of the $R C$ load on the inverter delay characteristics dominating the device parasitics, thus improving the accuracy of the transistor I-V model. This behavior also explains why the accuracy improves as the geometric size of the transistors and the parasitic device resistances and capacitances get smaller. Thus, these delay expressions become more accurate for higher $R C$ loads and more aggressive technologies.

\section{Power Estimation}

There are two primary contributions to the total power dissipated by a CMOS inverter, dynamic $C V^{2} f$ power dissipation and short-circuit power dissipation $[8,9]$. The logic stage following a large $R C$ load will dissipate significant amounts of short-circuit power due to the degraded waveform originating from the initial CMOS inverter. During the region where the input signal is between $V_{T N}$ and $V_{D D}+V_{T P}$, a $D C$ current path exists between $V_{D D}$ and ground. This excess current is called short-circuit (or crossover) current [9]. The short-circuit power dissipation of the following stage for one transition (either rising or falling edge) can be approximated by

$$
P_{S C}=\frac{1}{2} V_{D D} I_{\text {peak }} t_{t} f
$$

where $f$ is the frequency of operation, $t_{t}$ is the transition time of the input waveform, and $I_{\text {peak }}$ is the maximum saturation current of the load transistor. $I_{\text {peak }}$ depends on both $V_{G S}$ and $V_{D S}$, therefore it is both input waveform and load dependent. 
Table II. Estimate of short-circuit power dissipated by a CMOS inverter loading a CMOS inverter driving an $R C$ load (.8 $\mu \mathrm{m}$ CMOS technology).

\begin{tabular}{|c|c|c|c|c|}
\hline \multirow{2}{*}{$\begin{array}{c}\text { Load } \\
\text { Resistance }\end{array}$} & \multirow{2}{*}{$\begin{array}{c}\text { Load } \\
\text { Capacitance }\end{array}$} & \multicolumn{2}{|c|}{$\begin{array}{c}\text { Power }(\mu \mathrm{W}) \\
f=10 \mathrm{Mhz}\end{array}$} & \multirow{2}{*}{ Error } \\
\cline { 3 - 5 } & Analytic & SPICE & \\
\hline $10 \Omega$ & $.3 \mathrm{pF}$ & 1.70 & .99 & $42 \%$ \\
\hline $10 \Omega$ & $.5 \mathrm{pF}$ & 4.86 & 3.22 & $34 \%$ \\
\hline $10 \Omega$ & $1 \mathrm{pF}$ & 15.4 & 11.1 & $28 \%$ \\
\hline $100 \Omega$ & $.3 \mathrm{pF}$ & 2.09 & 1.23 & $41 \%$ \\
\hline $100 \Omega$ & $.5 \mathrm{pF}$ & 5.68 & 3.83 & $32 \%$ \\
\hline $100 \Omega$ & $1 \mathrm{pF}$ & 17.1 & 12.7 & $26 \%$ \\
\hline $1000 \Omega$ & $.3 \mathrm{pF}$ & 7.18 & 5.2 & $28 \%$ \\
\hline $1000 \Omega$ & $.5 \mathrm{pF}$ & 15.8 & 12.2 & $23 \%$ \\
\hline $1000 \Omega$ & $1 \mathrm{pF}$ & 41.7 & 3.38 & $19 \%$ \\
\hline
\end{tabular}

By inserting the transition time from (6) into (7), the short circuit power dissipation of a CMOS inverter following a lumped $R C$ load over both the rising and falling transitions is

$$
P_{S C}=2.3 V_{D D} I_{\text {peak }} f \frac{\frac{C}{K}+\mho_{d o} R C}{\mho_{d o}}
$$

The short-circuit power for a wide variety of $R C$ loads between the CMOS inverter stages derived from the analytical expression is compared to that of SPICE in Table II. For smaller loads, hence, faster transition times, there is neglible short circuit power since a direct path from the power supply to ground does not exist for any significant time. The short circuit power becomes non-neglible when larger interconnect loads between stages cause a transition time of significant magnitude, such as greater than $.5 \mathrm{~ns}$ for a $.8 \mu \mathrm{m}$ CMOS inverter. At this borderline value, SPICE departs from the analytical value by a maximum of $42 \%$. As the $R C$ load and transition time increase, the error decreases to less than $20 \%$. With the longer transition times, the analytically derived short circuit power closely approximates the short circuit power dissipation derived from SPICE. Furthermore, the short circuit power becomes significant when the CMOS inverter is loaded by larger $R C$ loads, creating long transition times. It is this condition that is of greatest interest when considering short circuit power in resistively loaded CMOS inverters.

\section{Conclusions}

A simple yet accurate expression for the output voltage of a CMOS inverter as a function of time driving a resistive-capacitive load is presented. With this expression, equations characterizing the propagation delay and transition time of a CMOS inverter driving an $R C$ load are presented. Furthermore, since the output waveform of this circuit is accurately modeled, the short-circuit power dissipation of the following CMOS stage loading the interconnect line can be estimated within $42 \%$, and typically under $20 \%$ for highly resistive lines. Therefore, due to the simplicity and accuracy of these expressions, the delay and power characteristics of a CMOS inverter driving a high impedance $R C$ interconnect line can be efficiently estimated.

\section{References}

[1] H. B. Bakoglu and J. D. Meindl, "Optimal Interconnection Circuits for VLSI," IEEE Transactions on Electron Devices, Vol. ED-32, No. 5, pp. 903-909, May 1985.

[2] S. Dhar and M. A. Franklin, "Optimum Buffer Circuits for Driving Long .Uniform Lines," IEEE Journal of Solid-State Circuits, Vol. SC-26, No. 1, pp. 32-40, January 1991.

[3] M. Nekili and Y. Savaria, "Optimal Methods of Driving Interconnections in VLSI Circuits," Proceedings of the IEEE International Symposium on Circuits and Systems, pp. 21-23, May 1992.

[4] C. Y. Wu and M. Shiau, "Delay Models and Speed Improvement Techniques for RC Tree Interconnections Among Small-Geometry CMOS Inverters," IEEE Journal of Solid-State Circuits, Vol. SC-25, No. 5, pp. 1247-1256, October 1990.

[5] A. I. Kayssi, K. A. Sakallah, and T. M. Burks, "Analytical Transient Response of CMOS Inverters," IEEE Transactions on Circuits and Systems-I, Vol. CAS I-39, No. 1, pp. 42-45, January 1992.

[6] T. Sakurai and A. R. Newton, "Alpha-Power Law MOSFET Model and its Applications to CMOS Inverter Delay and Other Formulas," IEEE Journal of Solid-State Circuits, Vol. SC-25, No. 2, pp. 584-594, April 1990.

[7] H. Shichman and D. A. Hodges, "Modeling and Simulation of Insulated-Gate Field-Effect Transistor Switching Circuits," IEEE Journal of Solid-State Circuits, Vol. SC-3, No. 3, pp. 285-289, September 1968.

[8] S. R. Vemuru and N. Scheinberg, "Short-Circuit Power Dissipation Estimation for CMOS Logic Gates," IEEE Transactions on Circuits and Systems-I: Fundamental Theory and Applications, Vol. CAS I-41, No. 11, pp. 762-766, November 1994.

[9] H. J. M. Veendrick, "Short-Circuit Dissipation of Static CMOS Circuitry and Its Impact on the Design of Buffer Circuits," IEEE Journal of Solid-State Circuits, Vol. SC-19, No. 4, pp. 468-473, August 1984. 